\title{
Atypical Manifestation of Gastroesophageal Reflux Disease: A Disease With a Thousand Faces
}

\author{
Tae Hoon Oh
}

Department of Internal Medicine, Sanggye Paik Hospital, Inje University College of Medicine, Seoul, Korea

Article: Relief of night-time symptoms associated with gastroesophageal reflux disease following 4 weeks of treatment with patoprazole magnesium: the Mexican gastroesophageal reflux disease working group López-Alvarenga JC, Orr W, Vagas-Romero JA, et al (J Neurogastroenterol Motil 2014;20:64-73)

The reflux of gastric content is more likely to present with troublesome symptoms rather than complications. Heartburn and acid regurgitation are widely regarded as the representative manifestations of gastroesophageal reflux disease (GERD) and are commonly referred to as typical symptoms, however pathologic acid reflux beyond esophagus can also result in a wide spectrum of GERD-related clinical presentations with or without typical symptoms. Hence, GERD-related disease manifestations are classified according to syndrome-based approach, reflecting that patient with GERD may present with symptom complex. ${ }^{1}$

In this issue of this journal, the Mexican GERD Working Group nicely reported the "Relief of night-time symptoms associated with gastroesophageal reflux disease following 4 weeks of treatment with pantoprazole-Mg: the Mexican gastroesophageal reflux disease working group." ${ }^{2}$ This was a large-scaled, nationwide, prospective, observational study enrolling 4,343 patients with typical symptoms plus at least one of other symptoms of GERD, recruited from more than 1,300 clinics in Mexico. After treatment of pantoprazole magnesium (pantoprazole- $\mathrm{Mg}$ ) once daily for 4 weeks, they evaluated the relief of wide range of GERD-related symptoms, especially focusing on night-time symptoms using physician's interview and patients completed questionnaire. Night-time GERD was defined as arousal from sleep during the night due to GERD-associated symptoms. Otherwise, they were defined as daytime GERD.

GERD can directly cause or be associated with a wide range of gastrointestinal symptoms. In this study, the authors investigated 16 GERD-related symptoms at baseline. As a result, 93\% of patients had more than 3 of symptoms while $7 \%$ of patients had all of 16 symptoms. And typical symptoms and concomitant atypical or extra-esophageal symptoms including chest pain, sleep disturbance, globus, chronic cough, dyspnea and dyspepsia symptoms i.e., epigastric pain, nausea, belching, early satiety, flatulence and retching were universally improved after 4 weeks of treatment with standard dose of pantoprazole-Mg. Although chronic cough, asthma and laryngopharyngitis are considered as

Received: November 30, 2013 Revised: December 5, 2013 Accepted: December 8, 2013

(c) This is an Open Access article distributed under the terms of the Creative Commons Attribution Non-Commercial License (http://creativecommons. org/licenses/by-nc/3.0) which permits unrestricted non-commercial use, distribution, and reproduction in any medium, provided the original work is properly cited.

*Correspondence: Tae Hoon Oh, MD, PhD

Division of Gastroenterology, Department of Internal Medicine, Inje University College of Medicine, Sanggye Paik Hospital, 1342, Dongil-ro, Nowon-gu, Seoul 139-707, Korea

Financial support: None.

Tel: +82-2-950-8867, Fax: +82-2-950-1955, E-mail: osbbang@paik.ac.kr

Conflicts of interest: None. 
extra-esophageal manifestation of GERD, therapeutic results of proton pump inhibitor (PPI) in patients with these symptoms have been conflicting. ${ }^{3}$ However, the results of this study confirmed that concomitant heartburn and/or regurgitation were reliable indicators to predict positive response to empirical trial of PPI therapy in patients with presumed extra-esophageal GERD, which was consistent with current recommendations. ${ }^{4}$ With regard to dose and duration of PPI therapy, although twice-daily PPI therapy for several months is primarily recommended in clinical practice based on several uncontrolled, open-labeled studies, ${ }^{5}$ this study suggests that standard dose for relatively shorter duration may be enough regimen to manage atypical symptoms if patients' chief complaint is typical symptom. ${ }^{4}$ Patients with GERD may also present with dyspepsia symptoms with or without typical reflux symptoms. In this study, dyspepsia symptoms were highly prevalent in up to $90 \%$ of patients and most of these symptoms were much improved after 4 weeks of PPI therapy. However, responsiveness to PPI therapy can not completely exclude a diagnosis of functional dyspepsia in which pathological acid reflux is not a mechanism for development of symptoms, because PPI test has a limited value in distinguishing between GERD patients with and without functional dyspepsia. ${ }^{6}$

Although the Montreal classification does not clearly classify the sleep disturbance as an extra-esophageal syndrome of GERD, nocturnal GERD is frequently associated with sleep disturbance. The survey of patients with GERD has reported that a prevalence of sleep disturbance attributed to typical symp-

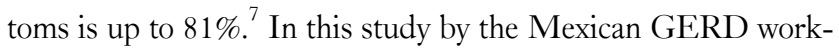
ing Group, there are some data supporting previous studies with regard to close association between nocturnal GERD and sleep disturbance. ${ }^{8}$ The prevalence of night-time GERD defined as arousal from sleep due to GERD-associated symptoms was $42.7 \%$ and that of sleep disturbance was $63.8 \%$. Although the detailed information regarding the symptom characteristics of sleep disturbance was not provided, $21.1 \%$ of patients who had sleep disturbance without arousal due to nocturnal GERD-associated symptoms might have "silent reflux" during night-time, since reflux at night may present with sleep disturbances without obvious symptom of heartburn. ${ }^{9}$ In addition, the authors have reported that, as compared with patients with daytime GERD, patients with night-time GERD were more likely to have extra-esophageal manifestations i.e., sleep disturbance, chronic cough and dyspnea. This finding is consistent with previous studies reporting that night-time heartburn is associated with more acid reflux in $\mathrm{pH}$ monitoring, the development of worse GERD-related symptoms, and higher prevalence of oropharyngeal, laryngeal and pulmonary manifestations. ${ }^{10-12}$ Although, in this study, endoscopic examination was not routinely performed, previous studies showed that the higher acid exposure in this subset of patients might lead to higher grade of esophagitis. ${ }^{13}$ Moreover, in this study, after 4 weeks of PPI therapy, better symptom relief in several atypical symptoms was found in patients with night-time GERD than those with daytime GERD. Based on this finding, the authors emphasized the effectiveness of pantoprazole- $\mathrm{Mg}$ in the relief of extra-esophageal symptoms especially related to night-time GERD. However, this study was not designed to directly compare the responsiveness of extra-esophageal symptoms to PPI therapy between night-time GERD and daytime GERD. Considering that night-time heartburn is associated with prolonged acid exposure resulting from physiologic changes of esophageal function during sleep and acid suppression naturally declines overnight when single dosage of PPI is taken before breakfast, extra-esophageal symptoms related to night-time GERD would be more likely to be resistant to PPI therapy. Further studies are needed to clarify this issue.

Aim of this study was to evaluate the effectiveness of pantoprazole-Mg in the relief of not only broad range of GERD symptoms but also in the night-time GERD-associated symptoms. However, there are several points to be considered, compared with other studies that have evaluated the efficacy of PPI therapy for the treatment of nocturnal heartburn and sleep disturbance. Patients enrolled in this study were heterogeneous group of GERD patients, not confined to patients with night-time GERD-related sleep disturbances. Moreover they might have had variable degree of nocturnal symptoms at baseline. In addition, nocturnal heartburn which has a high specific value for diagnosis of nocturnal GERD was not used as one of the efficacy parameters following PPI therapy. ${ }^{10}$ Lastly, the validated questionnaire for assessment of sleep quality, work productivity, and regular activity were not used.

In conclusion, significant number of patients with GERD may present with a wide range of atypical or extra-esophageal symptoms, which may be poorly recognized unless careful history-taking is achieved. Although it is difficult to establish a cause-and-effect association between GERD and atypical symptoms because of low specificity of diagnostic test, the concomitant presence of typical heartburn or regurgitation should guide to a provisional diagnosis of GERD. Prior to unnecessary investigation, empirical trial of PPI therapy would be an initial approach to find out the presumed mechanism. 


\section{References}

1. Vakil N, van Zanten SV, Kahrilas P, Dent J, Jones R; Global Consensus Group. The Montreal definition and classification of gastroesophageal reflux disease: a global evidence-based consensus. Am J Gastroenterol 2006;101:1900-1920.

2. López-Alvarenga JC, Orr W, Vagas-Romero JA, et al. Relief of night-time symptoms associated with gastroesophageal reflux disease following 4 weeks of treatment with patoprazole magnesium: the Mexican gastroesophageal reflux disease working group. J Neurogastroenterol Motil 2014;20:64-73.

3. Richter JE. Review article: extraoesophageal manifestations of gastro-oesophageal reflux disease. Aliment Pharmacol Ther 2005;22 (suppl 1):70-80.

4. Kahrilas PJ, Shaheen NJ, Vaezi MF, et al. American Gastroenterological Association Medical Position Statement on the management of gastroesophageal reflux disease. Gastroenterol 2008;135:1383-1391.

5. Ates F, Vaezi MF. Approach to the patient with presumed extraoesophageal GERD. Best Pract Res Clin Gastroenterol 2013;27: 415-431.

6. Xiao YL, Peng S, Tao J, et al. Prevalence and symptom pattern of pathologic esophageal acid reflux in patients with functional dyspepsia based on the Rome III criteria. Am J Gastroenterol 2010;105: 2626-2631.
7. Chand N, Johnson DA, Tabangin M, Ware JC. Sleep dysfunction in patients with gastro-oesophageal reflux disease: prevalence and response to GERD therapy, a pilot study. Aliment Pharmacol Ther 2004;20:969-974.

8. Jung HK, Choung RS, Talley NJ. Gastroesophageal reflux disease and sleep disorders: evidence for a causal link and therapeutic implications. J Neurogastroenterol Motil 2010;16:22-29.

9. Orr WC, Goodrich S, Fernström P, Hasselgren G. Occurrence of nighttime gastroesophageal reflux in disturbed and normal sleepers. Clin Gastroenterol Hepatol 2008;6:1099-1104.

10. Fornari F, Madalosso CA, Callegari-Jacques SM, Gurski RR. Heartburn during sleep: a clinical marker of gastro-oesophageal reflux disease in morbidly obese patients. Neurogastroenterol Motil 2009;21:136-142.

11. Dickman R, Green C, Fass SS, et al. Relationships between sleep quality and $\mathrm{pH}$ monitoring findings in persons with gastroesophageal reflux disease. J Clin Sleep Med 2007;3:505-513.

12. Dean BB, Aguilar D, Johnson LF, et al. Night-time and daytime atypical manifestations of gastro-oesophageal reflux disease: frequency, severity and impact on health-related quality of life. Aliment Pharmacol Ther 2008;27:327-337.

13. Adachi K, Fujishiro H, Katsube T, et al. Predominant nocturnal acid reflux in patients with Los Angeles grade C and D reflux esophagitis. J Gastroenterol Hepatol 2001;16:1191-1196. 